\title{
EEG power spectral analysis and saccadic eye movement for true and false information
}

\author{
Elvina Febriyani Chandrawijaya ${ }^{1}$, Ananta Yudiarso ${ }^{2}$, dan Yusti Probowati Rahayu ${ }^{3}$
}

\begin{abstract}
The purpose of this study was to examine the differences between the electroencephalography (EEG) power spectral analysis and saccadic eye movements (SEM)'s the response toward true and false information. The research method was conducted using an experimental design with true and false autobiographical information as stimuli. There were23 female participants aged between 19-24 years old $(M=21,45 ; S D=1,5)$. The results showed a significant difference in the number of SEM between false information and true information. The EEG power is greater in the frontal, temporal and central areas for false information. Interestingly, we found a correlation between EEG power and SEM in detecting true and false information. Our result findings indicated evidence of multimodality by combining electroencephalography (EEG) and saccadic eye movement (SEM) to dissociate false and true information responses. The distinct dissociative process between true and false information can be used for memory studies and developing lie detectors.
\end{abstract}

\section{Keywords}

Saccadic eye movement, electroencephalography, true information, false information

\section{Introduction}

True and false information mental processes are very important studies to detect information recognition. Information can be conveyed in the form of verbal communication or non-verbal communication. In general, verbal communication is a spoken or written communication, e.g., the use of words. Non-verbal communication does not include words but body language such as gestures, postures, facial expression, eye gaze, loudness or tone of voice, accent, etc (Mandal, 2014). A previous study shows that high school students in the sample reported, on average, 4.1 lied in the past 24 hours - a rate that was $75 \%$ higher than that reported by college students and $150 \%$ higher than that reported by the national sample of adults (Levine et al., 2013). Detecting lies in terms of true or false information has become a difficult task since there are no reliable verbal or nonverbal cues. Traditional lie detector methods applied skin conductivity to dissociate true and false information. However, these methods had been questioned for the bias of individual differences in stress responses. Therefore, (Vrij et al., 2010) encouraged further research on lying to be actively involved in discriminating different mental processes between honest and lying people. Accordingly, in this study, we suggest a study to detect true and false information. True information is the knowledge that is in accordance with the actual state or condition. False information is any knowledge that is not true or not recognized by the subjects. One alternative to detect true and false information is to use multimodality measurement in mental processing.

Cognitive process in true and false information can be observed from neural electrophysiology activities and saccadic eye movements. Combining the electroencephalography (EEG) and saccadic eye movement (SEM) can provide a comprehensive result to detect false and true information. Using EEG power, we can observe the electrophysiology activity during the true and false information stimulus. Previous research using SEM showed that the eye movements were related to memory retrieval (Nieuwenhuis et al., 2013). The use of multimodality of EEG and SEM was employed in this study. Both EEG and SEM could be a biomarker to dissociate mental processing between true and false information.

Combining EEG and SEM has been well researched in understanding perceptual and cognitive processing (Nikolaev et al., 2014), emotion (Simola et al., 2015), attention (Fischer et al., 2013), and memory (Nikolaev et al., 2011, 2014; Ohno et al., 2007). To our best knowledge, we rarely found studies that have combined EEG and SEM in recognizing true and false information.

\footnotetext{
1,2,3 Universitas Surabaya

Korespondensi:

Elvina Febriyani Chandrawijaya, Fakultas Psikologi Universitas Surabaya

Email: elvina.febriyani@gmail.com
} 
The available studies typically did not consider sexspecific brain differences. Therefore, we observed female subjects to control extraneous variable caused by brain structure differences in this study.

There were two reasons why we used EEG. Firstly, Electroencephalograph (EEG) can be used to see how our mental processes at real time. EEG measures the electrical activity of the brain at different sites of the head, typically using electrodes placed on the scalp (Freeman \& Quiroga, 2013). Secondly, EEG is a non-invasive measurement technique for brain activation patterns related to attention, learning, working memory, short-term memory, long-term memory (Amin et al., 2014) and autobiographical memory

(Imperatori et al., 2014). EEG power has been used to study brain regions that were responsible for memory functions (Chen et al., 2006). True and false information involved the memory process.

In this study, we used EEG spectral analysis using EEG power. EEG power represents the amount of activity in certain frequency bands of the signal while the coherence between different electrodes reflects the degree to which connections are present across brain regions (Xiao et al., 2018). The intensity of power at electrodes in certain frequency bands is measured in $\mathrm{V} 2$.

Power spectral analysis is an accepted method used for analyzing the EEG signal in the field of neuroscience. Electroencephalogram (EEG) spectral analysis quantifies the amount of rhythmic (or oscillatory) activity of different frequency in EEGs (Im, 2018). The power spectrum is analysed on the basis of broader frequency bands, which represent the sum of power of several smaller frequency bands (Dressler et al., 2004). In the present study, we examined which parts of the EEG power spectrum were most active or had higher voltage for discrimination between true information and false information recognition.

True and false information process involved memory. Memory is the capacity to store and retrieve information (Zlotnik \& Vansintjan, 2019). Memory covers three important aspects of information processing. Firstly, memory encoding is where we change the input from sensory system to visual, acoustic or sematic code. After that we store the memory at short term memory (STM) or long-term memory (LTM). STM can store information for 30 seconds but LTM can last a lifetime. Lastly, memory retrieval is when we get the information out of storage (McLeod, 2013). One example of LTM is autobiographical memory. Autobiographical memory (AM) is the ability to remember information from one's own life. It is a dynamic integration of episodic memory (emotional images) and semantic memory (knowledge of facts) (Chen et al., 2017). We examine oscillations in EEG to understand how people retrieve memory from the long-term memory and how they recognize information.

EEG signals are easily contaminated by external sources. These "artifacts," inherent of scalp EEG recordings, are produced by head movements, blinking, electrocardiogram, muscle activity, etc (Freeman \& Quiroga, 2013). Therefore, we were anticipating another nonverbal cue that might not interfere with EEG result and cause minimal artifacts. Recently, the co-registration of eye movements and EEG has been done to study memory encoding (Nikolaev et al., 2011, 2013). However, the eye movement might produce artefact. Therefore, the general strategy to remove artifacts is by asking the participant to avoid head movements and eye blinking during the experiment. Another alternative, a common practice to avoid artifacts from eye movements is to measure epochs before and after the eye movements. Nevertheless, some artifacts can be extended before and after the saccades and the analysis of that period may be confounded (Plöchl et al., 2012). In addition, by attempting to remove eye movement artifacts, subjects are usually required not to move their eyes. This adds an unnatural cognitive load to the experimental task and creates unnatural viewing conditions.

Saccades are fast ballistic ocular muscle movements that are executed about three times per second and bring the gaze from one point to another (Graupner et al., 2011). This ocular movement happens when one is engaged in tasks related to the search of information in the long-term memory. SEM is also related to memory enhancement and it was tested across various materials and test situations (Parker \& Dagnall, 2012). Bilateral (right-left) SEM increased the interaction between the brain's hemispheres and the activation of memory retrieval (Nieuwenhuis et al., 2013; Parker \& Dagnall, 2012). Previous researches had been held using SEM for lie detectors (Borza et al., 2018; Lim et al., 2013; Vrij et al., 2015). A person that is lying would show more saccades compared to a person telling the truth (Vrij et al., 2015). This research would not study about active lying but information recognition process. This research would try to reveal the dynamics of cognitive processing between true and false information that could be used to support cognitive theories in studies about lie detectors.

In this study, the EEG and SEM dynamics during memory recall and the brain regions that discriminate true and false information were explored. The true and false information presented in this study was about the autobiographical memory. Another reason to research EEG spectral analysis and SEM for true and false information processes was to test the effectiveness of combining EEG power spectral analysis and SEM. Previous researches had been held using event related potential (ERP) and SEM (Fischer et al., 2013; Frey et al., 2013; Nikolaev et al., 2013). ERP describes the changes in brain activities in relation to a certain situation (Chen et al., 2018). ERPs which are recorded from the central nervous system are related to recognition of notable events. However, it is very difficult to distinguish the single-trial ERPs due to their low amplitude and their similarity to spontaneous fluctuations in the EEG (Freeman \& Quiroga, 2013). Whereas, the EEG power is representing the activity amount in certain 
frequency bands that reflects brain electrical activity across brain regions (Nunez \& Srinivasan, 2009). EEG power and SEM data analysis were combined for multimodality measurement. These two modalities are difficult to measure because SEM can cause artifacts in EEG power. In this study, we observed at the relationship between these two modalities in recognizing true and false information.

\section{Metode}

\section{Participants}

There are important differences that distinguish the male from the female brain (Cosgrove et al., 2007). Therefore, in this study we would only focus on female participants. Twenty-three females (aged between 18 to 26 years old $(M=21,45 ; \mathrm{SD}=1,5)$ who fulfilled the chosen criteria took part in this research. To increase the internal validity of the measurement of EEG and SEM and to avoid extraneous variables, we used a certain inclusion criterion. The inclusion criterion were females with no suffer from epilepsy, brain trauma, and head injury. The subjects were also not consuming any medication or having neurological disorders.

\section{Design}

Within subject experimental design was employed in this research to measure EEG and SEM during two conditions of visual true and false stimulus.

\section{Procedure}

Participants filled out an informed consent form related to their willingness to participate in this research and were then asked to give autobiographical data that would then be presented as stimulus. Stimulus were presented with a passive oddball paradigm. We developed two types of stimulus true information and false information regarding participants' autobiography (e.g., names, address, family name, date of birth, place of birth). False information was irrelevant stimuli to the participant or incorrect information. In this experimental paradigm, the number of false and true stimuli were the same numbers and durations. Each stimulus was exposed to the participants for 5 seconds. The participants were asked not to give any responses and minimize any movements during the treatment to avoid the artifacts. Participants were instructed to lay down and to minimize any muscular movement including the blinking of the eyes. Instruments used were EEG and a professional quality video recorder with WVGA-120 resolutions recording SEM activities. Participants were then rigged to 19 channel EEG electrodes according to the International Electrode Placement System which entailed 10-20 on the scalp in lay down position. The 19 EEG channels were used including frontal pole (Fp1, Fp2), frontal (F3, F7, Fz, F4, F8), central temporal (C3, Cz, C4), temporal (T3, T4, T5, T6), parietal (P3, Pz, $\mathrm{P} 4)$, and occipital $(\mathrm{O} 1, \mathrm{O} 2)$. The artifact of EEG power had been controlled by a statistical method using principal component analysis.

\section{Data Analysis}

The EEG spectral brain was analyzed using the Profusion EEG program. This process resulted in EEG brain power and EEG brain map. We defined spectral analysis using four band frequencies including beta $(>13 \mathrm{~Hz})$, alpha $(8-$ $13 \mathrm{~Hz})$, theta $(4-8 \mathrm{~Hz})$, and delta $(0.5-4 \mathrm{~Hz})$. We analysed the factor loading of each EEG power with principal component analysis to control artifacts. Eye movements were recorded using a video recorder. The visual data were analyzed by three raters (graders) to count the frequency of saccadic eye movements in false and true stimuli conditions. The visual data of SEM were analyzed in a slow-motion recording. Intraclass Correlation Coefficient (ICC) was used to measure the reliability of the raters' (graders') measurement. Parametric and non-parametric statistical analysis were used to verify the differences of EEG brain power for each band frequency and the number of SEM occurred between true and false information stimuli.

\section{Result}

The result of reliability test between raters indicated high reliability. Intraclass correlation coefficient between raters showed quite satisfactory results $(\mathrm{rxx}=0.925)$. ANOVA analysis showed that there was no difference between raters with a significance value of $0.234(p 0.05)$.

We found a significant difference of SEM between two conditions $(\mathrm{p}=0.001)$. Mean of true information= $3,47(\mathrm{SD}=1,83)$ and mean of false information $=4,94$ $(\mathrm{SD}=2,34)$ (Figure 1).

The principal component analysis showed strong to moderate varimax factor values for EEG power in most of all frequency bands. The artifacts from eye movements did not confound the EEG power data in all frequency bands.

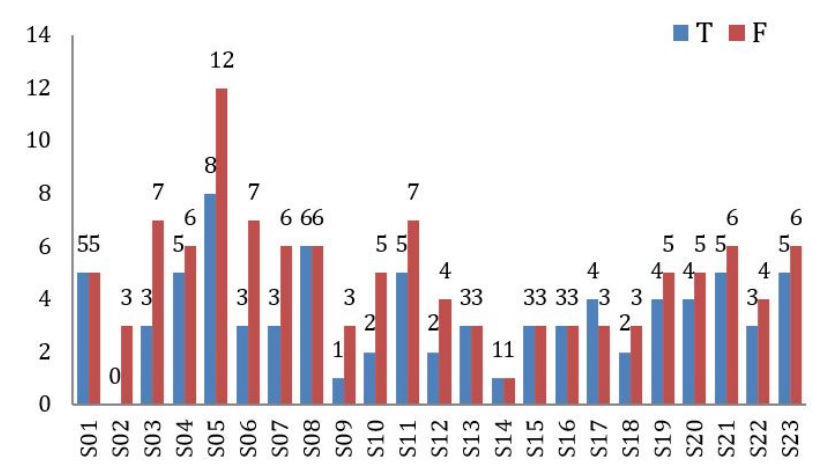

Figure 1. Comparison of SEM total amounts on $\mathrm{T}$ and $\mathrm{F}$ conditions. 


\section{Different brain activity between true and false information}

Our result found a significant difference in EEG brain power in delta waves (T5), alpha waves (F3 \& Fz) and beta waves (F3 \& C3) between true and false condition (Table 2).

The left temporal lobe (T5) is the center of verbal understanding and memory since it is located in the hippocampus. Hippocampus is an area of the brain specifically related to LTM (Yonelinas, 2013). The stimulus presented in this research was autobiographical information and thus needed a verbal ability to process

Table 1. Principal Component Analysis Results

\begin{tabular}{|c|c|c|c|c|c|}
\hline \multicolumn{6}{|c|}{ True Information } \\
\hline Chanel & & Delta & Theta & Beta & Alpha \\
\hline $\mathrm{Fp} 1$ & & 0,714 & 0,524 & 0,670 & 0,707 \\
\hline Fp2 & & 0,665 & 0,572 & 0,756 & 0,808 \\
\hline F3 & & 0,843 & 0,811 & 0,613 & 0,672 \\
\hline $\mathrm{F} 4$ & & 0,805 & 0,816 & 0,706 & 0,737 \\
\hline C3 & & 0,850 & 0,873 & 0,348 & 0,834 \\
\hline C4 & & 0,617 & 0,845 & 0,841 & 0,928 \\
\hline P3 & & 0,908 & 0,890 & 0,875 & 0,946 \\
\hline P4 & & 0,157 & 0,872 & 0,772 & 0,916 \\
\hline O1 & & 0,803 & 0,830 & 0,813 & 0,877 \\
\hline $\mathrm{O} 2$ & & 0,580 & 0,835 & 0,771 & 0,888 \\
\hline F7 & & 0,675 & 0,836 & 0,705 & 0,836 \\
\hline F8 & & 0,664 & 0,888 & 0,888 & 0,928 \\
\hline T3 & & 0,838 & 0,845 & 0,567 & 0,642 \\
\hline T4 & & 0,702 & 0,907 & 0,578 & 0,764 \\
\hline T5 & & 0,793 & 0,783 & 0,764 & 0,860 \\
\hline T6 & & 0,891 & 0,875 & 0,772 & 0,913 \\
\hline $\mathrm{Fz}$ & & 0,557 & 0,687 & 0,725 & 0,703 \\
\hline $\mathrm{Cz}$ & & 0,810 & 0,811 & 0,793 & 0,767 \\
\hline $\mathrm{Pz}$ & & 0,891 & 0,898 & 0,861 & 0,929 \\
\hline \multicolumn{6}{|c|}{ False Information } \\
\hline Chanel & & Delta & Theta & Beta & Alpha \\
\hline$\overline{F p 1}$ & & 0,740 & 0,452 & 0,646 & 0,775 \\
\hline Fp2 & & 0,754 & 0,513 & 0,834 & 0,846 \\
\hline F3 & & 0,692 & 0,788 & 0,736 & 0,673 \\
\hline $\mathrm{F} 4$ & & 0,713 & 0,792 & 0,830 & 0,840 \\
\hline C3 & & 0,686 & 0,779 & 0,471 & 0,711 \\
\hline C4 & & 0,599 & 0,822 & 0,917 & 0,945 \\
\hline P3 & & 0,858 & 0,731 & 0,935 & 0,917 \\
\hline $\mathrm{P} 4$ & & 0,123 & 0,644 & 0,920 & 0,824 \\
\hline O1 & & 0,924 & 0,552 & 0,893 & 0,872 \\
\hline $\mathrm{O} 2$ & & 0,782 & 0,588 & 0,909 & 0,852 \\
\hline F7 & & 0,711 & 0,802 & 0,664 & 0,788 \\
\hline F8 & & 0,560 & 0,929 & 0,847 & 0,903 \\
\hline T3 & & 0,775 & 0,707 & 0,556 & 0,689 \\
\hline T4 & & 0,872 & 0,746 & 0,731 & 0,768 \\
\hline T5 & & 0,889 & 0,665 & 0,855 & 0,897 \\
\hline T6 & & 0,835 & 0,704 & 0,916 & 0,862 \\
\hline $\mathrm{Fz}$ & & 0,629 & 0,656 & 0,734 & 0,716 \\
\hline $\mathrm{Cz}$ & & 0,737 & 0,776 & 0,774 & 0,750 \\
\hline $\mathrm{Pz}$ & & 0,857 & 0,652 & 0,919 & 0,921 \\
\hline Total & e & 54.028 & 58.514 & 59.651 & 212 \\
\hline
\end{tabular}

and memory to retrieve from the LTM. Delta waves that produced in the waking state will provide an opportunity to access the subconscious activity, encourage the flow into conscious thought (Wan Ismail et al., 2016). This is related to the retrieval process of long-term memory as LTM is often outside the conscious mind

Theta waves $(4 \mathrm{hz}-8 \mathrm{hz})$ are a wavelength that occur when a person is in deep relaxation, meditation. On this research, the participant was concentrating on the task thus showing no significant difference on EEG power on true and false conditions of this research.

On alpha waves (8-13 hz), the significant difference was at F3 \& Fz channel with a significance value of $0.011(\mathrm{p}<0.05)$ and $0.016(\mathrm{p}<0.05) . F 3$ is the center of motor planning while $\mathrm{Fz}$ is related to working memory. On this wavelength, a person is in a state of calm and consciousness. Alpha brain waves are ideal condition for reflection, problem solving and visualization of creativity (Wan Ismail et al., 2016). The frontal lobe is responsible for higher cognitive functions such as memory, language, attention, and emotion (Hoffmann, 2013). The stimulus given is related to autobiographical memory causing EEG power generated in this area to be bigger on true conditions. When someone recognizes a piece of information, EEG power will be bigger on the frontal lobe.

Beta waves $(>13 \mathrm{~Hz})$ occurred when we focused and concentrated on a task. Beta waves are beneficial for activities that require high alertness. The areas showing a significant difference were F3 and C3. F3 is related to attention and the thinking process, while $\mathrm{C} 3$ is related to motor integration of the body parts. Sensor-motor integration is the ability for the central nervous system (CNS) to unite many sources of stimulus beginning with sensory input, then processing that input into a motoric movement. In this research, the significant difference on C3 can be attributed to the appearance of SEM related to recognition of true and false information. Therefore, F3 and C3 channels showed significant differences in EEG power on true and false conditions.

As shown in Figure 2, there are differences in EEG spectral analysis in response to true and false information. In Figur 2(A), the dominant area of the

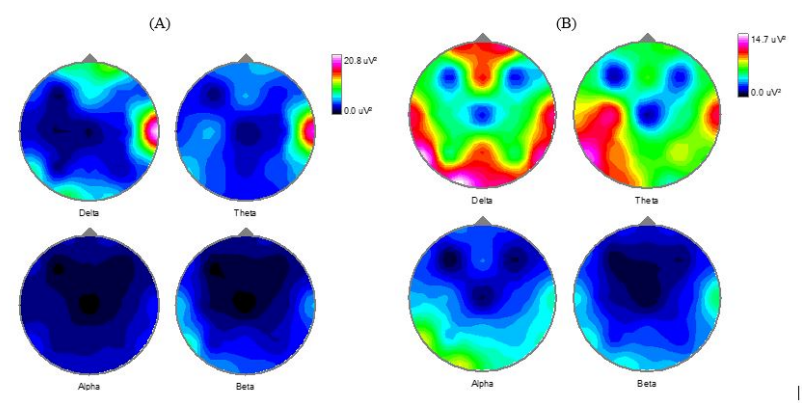

Figure 2. Typical patterns of topographic maps of EEG brain power for all frequency band corresponding to true (a) and false information (b). 
Table 2. Wilcoxon Hypothesis Test Result On EEG Power Data

\begin{tabular}{|c|c|c|c|c|c|c|c|c|}
\hline \multirow[b]{3}{*}{ Channel } & \multicolumn{8}{|c|}{ Band Frequencies } \\
\hline & \multicolumn{2}{|c|}{ Delta } & \multicolumn{2}{|c|}{ Theta } & \multicolumn{2}{|c|}{ Alpha } & \multicolumn{2}{|c|}{ Beta } \\
\hline & Z & Sig & Z & Sig & Z & Sig & Z & Sig \\
\hline Fp1 & -0.700 & 0.484 & -0.487 & 0.627 & -1.642 & 0.101 & -0.122 & 0.903 \\
\hline Fp2 & -0.152 & 0.879 & -0.030 & 0.976 & -0.182 & 0.855 & -0.091 & 0.927 \\
\hline F3 & -0.152 & 0.879 & -0.700 & 0.484 & -2.555 & 0.011 & -2.494 & 0.013 \\
\hline $\mathrm{F} 4$ & -0.152 & 0.879 & -0.517 & 0.605 & -0.669 & 0.503 & -1.399 & 0.162 \\
\hline C3 & -1.277 & 0.201 & -0.304 & 0.761 & -1.308 & 0.191 & -2.251 & 0.024 \\
\hline C4 & -0.852 & 0.394 & -0.547 & 0.584 & -0.030 & 0.976 & -1.825 & 0.068 \\
\hline P3 & -1.642 & 0.101 & -0.122 & 0.903 & -0.669 & 0.503 & -1.703 & 0.089 \\
\hline P4 & -1.551 & 0.121 & -1.460 & 0.144 & 0.000 & 1.000 & -1.794 & 0.073 \\
\hline 01 & * & * & -0.213 & 0.831 & -1.095 & 0.274 & -1.521 & 0.128 \\
\hline $\mathrm{O} 2$ & -1.673 & 0.094 & -1.217 & 0.224 & -0.882 & 0.378 & -1.034 & 0.301 \\
\hline F7 & -1.217 & 0.224 & -0.669 & 0.503 & -1.338 & 0.181 & -1.308 & 0.191 \\
\hline F8 & -1.673 & 0.094 & -0.882 & 0.378 & -0.608 & 0.543 & -1.034 & 0.301 \\
\hline T3 & -1.095 & 0.274 & -0.274 & 0.784 & * & * & * & * \\
\hline T4 & -1.034 & 0.301 & -0.426 & 0.670 & -0.912 & 0.362 & -1.582 & 0.114 \\
\hline T5 & -2.038 & 0.042 & -0.122 & 0.903 & -0.882 & 0.378 & -0.852 & 0.394 \\
\hline T6 & -1.764 & 0.078 & -0.608 & 0.543 & -0.547 & 0.584 & -1.551 & 0.121 \\
\hline $\mathrm{Fz}$ & -1.703 & 0.089 & -0.274 & 0.784 & -2.403 & 0.016 & -1.734 & 0.083 \\
\hline $\mathrm{Cz}$ & -1.430 & 0.153 & -1.065 & 0.287 & -0.24 & 0.808 & -1.217 & 0.224 \\
\hline $\mathrm{Pz}$ & -1.430 & 0.153 & -0.426 & 0.670 & -0.852 & 0.394 & -1.369 & 0.171 \\
\hline
\end{tabular}

${ }^{*}$ Tested with the parametric hypothesis test (paired sample t-test).

delta power was observed on the temporal region at first and then extended across frontal area. Temporal lobe is associated with auditory, olfactory, vestibular, visual senses and the perception of spoken and written language (Kiernan, 2012). When processing the visual and written autobiographical stimuli, the temporal area was activated and it was associated with increased power in that area. As shown in Fig. 2b, the dominant area of delta power was observed on temporal, frontal and occipital region at first and then extended across central and parietal area. The difference between true and false stimulus brain topographic maps in delta band shows a pattern of increased lateralization or activity in the two hemispheres in response to the false stimuli. This result was in line with the correlation result between EEG brain power and SEM that has a tendency to lateralize more in response to false stimuli.

\section{Correlation between EEG Power and SEM}

We found a significant correlation between SEM and EEG power on true and false conditions (Table 3). In false information stimuli, there were significant correlations among SEM and EEG channels located at the frontal (F3, F4), parietal (P4), central (C4, Cz), Occipital (O1) and temporal (T3, T5). While in true information stimuli, there were significant correlations among SEM and EEG channels located at the parietal (P3, P4, Pz), central (C4), occipital (O2) and temporal (T3, T4). Significant correlations among EEG brain power and SEM were found mostly at delta waves. In the cognitive domain, the delta wave was involved in attention, salience detection, and perception (Knyazev, 2012).
In the theta waves, there was no significant correlation between EEG power and SEM during a true condition. In a false condition the correlation was found in frontal and temporal lobe. In alpha waves, there was a significant correlation between EEG and SEM at frontal lobe in the true condition but in the false condition there was no correlation in frontal area of the brain. In beta waves, there was a significant correlation between EEG power and SEM at parietal lobe during the true condition but not in the false condition. From this result we concluded that if someone recognizes true information, there will be no significant correlation between EEG power and SEM frequency in frontal area delta waves. In contrast, if someone recognizes false information, there will be a significant correlation between EEG power and SEM frequency in frontal area delta waves.

\section{Discussions}

In this research we found some positive findings. Firstly, there were significant differences in EEG power and secondly there were correlations between SEM and EEG brain power between true and false information processing. Our results supported cognitive load theory. False information would have more cognitive load and exhibit an increased number of SEM (Vrij et al., 2015). The true information on long-term memory exhibits less saccadic eye movement (Nieuwenhuis et al., 2013; Parker \& Dagnall, 2012).

This research indicated the difference between areas of the active brain on true and false conditions, specifically the frontal area. The frontal area's processed memory and 
Table 3. The Correlation Between EEG Brain Power And SEM Frequency During True And False Condition

\begin{tabular}{|c|c|c|c|c|c|c|c|c|}
\hline & \multicolumn{8}{|c|}{ True } \\
\hline & \multicolumn{2}{|c|}{ Delta } & \multicolumn{2}{|c|}{ Theta } & \multicolumn{2}{|c|}{ Alpha } & \multicolumn{2}{|c|}{ Beta } \\
\hline & $R$ & Sig. & $\mathrm{R}$ & Sig. & $r$ & Sig. & $r$ & Sig. \\
\hline Fp1 & 0.099 & 0.533 & 0.254 & 0.110 & 0.167 & 0.291 & 0.021 & 0.892 \\
\hline Fp2 & 0.116 & 0.465 & 0.228 & 0.151 & 0.270 & 0.088 & 0.262 & 0.099 \\
\hline F3 & 0.202 & 0.203 & 0.245 & 0.123 & 0.167 & 0.291 & 0.124 & 0.432 \\
\hline $\mathrm{F} 4$ & 0.202 & 0.203 & 0.176 & 0.267 & 0.347 & 0.028 & 0.150 & 0.343 \\
\hline C3 & 0.287 & 0.070 & 0.108 & 0.498 & 0.124 & 0.432 & -0.004 & 0.978 \\
\hline C4 & 0.356 & 0.025 & 0.194 & 0.223 & 0.210 & 0.185 & 0.124 & 0.432 \\
\hline P3 & 0.347 & 0.028 & 0.220 & 0.167 & 0.313 & 0.048 & 0.253 & 0.110 \\
\hline P4 & 0.313 & 0.048 & 0.151 & 0.343 & 0.304 & 0.055 & 0.322 & 0.042 \\
\hline 01 & 0.244 & 0.123 & 0.185 & 0.244 & 0.296 & 0.062 & 0.167 & 0.291 \\
\hline $\mathrm{O} 2$ & 0.322 & 0.042 & 0.125 & 0.432 & 0.210 & 0.185 & 0.253 & 0.110 \\
\hline F7 & 0.244 & 0.123 & 0.022 & 0.892 & -0.090 & 0.570 & -0.116 & 0.465 \\
\hline F8 & 0.133 & 0.401 & 0.271 & 0.088 & 0.262 & 0.099 & 0.081 & 0.607 \\
\hline T3 & 0.330 & 0.037 & 0.176 & 0.267 & 0.184 & 0.244 & 0.064 & 0.685 \\
\hline T4 & 0.322 & 0.042 & 0.194 & 0.223 & 0.116 & 0.465 & 0.021 & 0.892 \\
\hline T5 & 0.296 & 0.062 & 0.133 & 0.401 & 0.081 & 0.607 & 0.090 & 0.570 \\
\hline T6 & 0.270 & 0.088 & 0.056 & 0.725 & 0.176 & 0.267 & 0.133 & 0.401 \\
\hline $\mathrm{Fz}$ & 0.253 & 0.110 & 0.220 & 0.167 & 0.339 & 0.032 & 0.339 & 0.032 \\
\hline $\mathrm{Cz}$ & 0.296 & 0.062 & 0.176 & 0.267 & 0.193 & 0.223 & 0.227 & 0.151 \\
\hline \multirow[t]{4}{*}{$\mathrm{Pz}$} & 0.339 & 0.032 & 0.133 & 0.401 & 0.219 & 0.167 & 0.202 & 0.203 \\
\hline & \multicolumn{8}{|c|}{ False } \\
\hline & \multicolumn{2}{|c|}{ Delta } & \multicolumn{2}{|c|}{ Theta } & \multicolumn{2}{|c|}{ Alpha } & \multicolumn{2}{|c|}{ Beta } \\
\hline & $R$ & Sig. & $R$ & Sig. & $r$ & Sig. & $r$ & Sig. \\
\hline Fp1 & 0.104 & 0.515 & 0.294 & 0.065 & 0.138 & 0.385 & 0.112 & 0.481 \\
\hline $\mathrm{Fp} 2$ & 0.086 & 0.588 & 0.294 & 0.065 & 0.147 & 0.356 & 0.216 & 0.175 \\
\hline F3 & 0.320 & 0.045 & 0.302 & 0.058 & 0.216 & 0.175 & 0.233 & 0.143 \\
\hline $\mathrm{F} 4$ & 0.328 & 0.039 & 0.268 & 0.093 & 0.250 & 0.116 & 0.268 & 0.093 \\
\hline C3 & 0.086 & 0.588 & 0.104 & 0.515 & 0.181 & 0.255 & 0.086 & 0.588 \\
\hline C4 & 0.406 & 0.011 & 0.216 & 0.175 & 0.233 & 0.143 & 0.225 & 0.158 \\
\hline P3 & 0.311 & 0.051 & 0.276 & 0.083 & 0.320 & 0.045 & 0.250 & 0.116 \\
\hline P4 & 0.389 & 0.015 & 0.181 & 0.255 & 0.250 & 0.116 & 0.259 & 0.104 \\
\hline 01 & 0.328 & 0.039 & 0.294 & 0.065 & 0.363 & 0.023 & 0.233 & 0.143 \\
\hline $\mathrm{O} 2$ & 0.242 & 0.129 & 0.207 & 0.193 & 0.294 & 0.065 & 0.259 & 0.104 \\
\hline F7 & 0.259 & 0.104 & 0.216 & 0.175 & 0.052 & 0.745 & -0.035 & 0.828 \\
\hline F8 & 0.104 & 0.515 & 0.320 & 0.045 & 0.207 & 0.193 & 0.199 & 0.212 \\
\hline T3 & 0.354 & 0.026 & 0.397 & 0.013 & 0.259 & 0.104 & 0.216 & 0.175 \\
\hline T4 & 0.311 & 0.051 & 0.242 & 0.129 & 0.155 & 0.329 & 0.086 & 0.588 \\
\hline T5 & 0.328 & 0.039 & 0.294 & 0.065 & 0.311 & 0.051 & 0.164 & 0.303 \\
\hline T6 & 0.294 & 0.065 & 0.242 & 0.129 & 0.268 & 0.093 & 0.225 & 0.158 \\
\hline $\mathrm{Fz}$ & 0.294 & 0.065 & 0.268 & 0.093 & 0.268 & 0.093 & 0.337 & 0.034 \\
\hline $\mathrm{Cz}$ & 0.337 & 0.034 & 0.276 & 0.083 & 0.285 & 0.073 & 0.225 & 0.158 \\
\hline $\mathrm{Pz}$ & 0.311 & 0.051 & 0.190 & 0.233 & 0.311 & 0.051 & 0.294 & 0.065 \\
\hline
\end{tabular}

deliberate creation of saccades. Within the frontal cortex, the cortical structures of the saccadic systems include; the frontal eye field (FEF) and its role in saccade and pursuit eye movement control, the supplementary eye field (SEF) and the dorsolateral prefrontal cortex (dlPFC) (Pouget, 2015). These three main areas involved in saccades are located in frontal lobe. When a person is thinking, the information processing in working memory causes the frontal cortex area to become more active and increase SEM frequency. SEM increases the interaction between hemispheres via the corpus callosum which in turn increases memory retrieval on the LTM. When participant recognizes a piece of incorrect information, they will need a more complex thought process to cross-check data and facts from the LTM. This explained why there was no significant correlation in true conditions between EEG and SEM results on the frontal lobe.

On delta waves, the largest correlation was found on the C4 channel between SEM and EEG on false conditions. $\mathrm{C} 4$ (central sulcus) is directly related with sensory-motoric integration. (Velasques et al., 2011) in previous research stated that SEM is directly related to the attention processes 
by uniting various visual information with oculomotor movements which was considered to be the first step of sensorimotor integration. A strong correlation was found between SEM and EEG power on the C4 channel.

\section{Conclusion \& Implication}

This research has come up with three findings. Firstly, there was a significant difference on the amount of SEM between true and false information. False information produces more SEM compared to true information. Secondly, there was a significant difference in EEG power when someone recognized true and false autobiography information on the temporal (T5) area in delta waves and on the frontal (F3 and FZ) and central (C3) in alpha and beta waves. Lastly, there was a significant relation between SEM and EEG. In false conditions, the frontal correlated significantly.

There were two implications of our findings. Firstly, our findings support distinct dissociative processes between true and false information. These processes can be detected by SEM and EEG brain power. Secondly, the correlations between EEG brain power and SEM supported a detection system for true and false information. Both can be developed for basic multi modalities for a lie detector. EEG artefacts, manual calculation of SEM, and small sample size of study were our main weaknesses in this research. Future research worth replicating this study.

Further research on non-spontaneous lies or trained lies is suggested. In this research, researchers used stimulus related to memory recall that does not involve emotions. Future research can use stimulus related to emotions to support various emotion theories related to lies and deceptions. This research only examined the cognitive aspect of information recognition and ignored emotions when in reality a lie can be related or elicit an emotion. Emotions affect the electrophysiological response of the brain and the biological response of the body. Future research can use the biological response related to emotion to map out its correlation to various specific parts of the brain. Brain imaging techniques can be done using other devices such as fMRI, CAT or PET. Furthermore, the result of this research can only be applied on recognition of specific information related to autobiographical information. The research finding of this research is still unable to reach deeper into the complex questions related to criminality. Detecting deception on real-life conditions requires a mix of both verbal and nonverbal techniques. This research focused solely on nonverbal responses measured using EEG and SEM. In reality, questioning techniques and question's characteristics are also important factors in lie detection. Future research can focus more on verbal detection of deceptions or use both verbal and non-verbal techniques at the same time.

\section{References}

Amin, H. U., Malik, A. S., Badruddin, N., \& Chooi, W. T. (2014). Brain behavior in learning and memory recall process: A high-resolution EEG analysis. IFMBE Proceedings, 43, 683-686. https://doi.org/10.1007/978-3-319-02913-9_174

Borza, D., Itu, R., \& Danescu, R. (2018). In the eye of the deceiver: Analyzing eye movements as a cue to deception. Journal of Imaging, 4(10). https://doi.org/10. 3390/jimaging4100120

Chen, H. Y., Gilmore, A. W., Nelson, S. M., \& McDermott, K. B. (2017). Are there multiple kinds of episodic memory? An fMRI investigation comparing autobiographical and recognition memory tasks. Journal of Neuroscience, 37(10).27642775. https://doi.org/10.1523/JNEUROSCI.1534-16.2017

Chen, Y., Patel, V. M., Phillips, P. J., Chellappa, R., Poon, T. W. K., Friesen, M. R., Wang, X., Li, X., Leung, V. C. M., Shukla, S., Yadav, R. N., Zorzi, M., Zanella, A., Testolin, A., Grazia, M. D. F. de, Zorzi, M., Guo, L., Jin, B., Yu, R., ... Kose, U. (2018). An optimizing and differentially private clustering algorithm for mixed data in sdn-based smart grid. IEEE Access, 6. https://doi.org/10.1109/ACCESS.2019.2909048

Chen, K.H., Wu, C.H. and Yao, G. (2006) Applicability of the WHOQoL-BREF on early adolescence. Social Indicators Research, 79, 215-234. https://doi.org/10.1007/s11205-0050211-0

Cosgrove, K. P., Mazure, C. M., \& Staley, J. K. (2007). Evolving knowledge of sex differences in brain structure, function, and chemistry. Biological psychiatry, 62(8), 847-855. https: //doi.org/10.1016/j.biopsych.2007.03.001

Dressler, O., Schneider, G., Stockmanns, G., \& Kochs, E. F. (2004). Awareness and the EEG power spectrum: Analysis of frequencies. British Journal of Anaesthesia, 93(6). https: //doi.org/10.1093/bja/aeh270

Fischer, T., Graupner, S.-T., Velichkovsky, B. M., \& Pannasch, S. (2013). Attentional dynamics during free picture viewing: Evidence from oculomotor behavior and electrocortical activity. Frontiers in Systems Neuroscience, 7. https://doi.org/ 10.3389/fnsys.2013.00017

Freeman, W.J. \& Quiroga, R.Q. (2013) Imaging brain function with EEG: Advanced temporal and spatial analysis of electroencephalographic signals, New York: Springer.

Frey, A., Ionescu, G., Lemaire, B., López-Orozco, F., Baccino, T., \& Guérin-Dugué, A. (2013). Decision-making in information seeking on texts: an eye-fixation-related potentials investigation. Frontiers in systems neuroscience, 7, 39. https: //doi.org/10.3389/fnsys.2013.00039

Graupner, S. T., Pannasch, S., \& Velichkovsky, B. M. (2011). Saccadic context indicates information processing within visual fixations: Evidence from event-related potentials and eye-movements analysis of the distractor effect. International Journal of Psychophysiology, 80(1). https:// doi.org/10.1016/j.ijpsycho.2011.01.013

Hoffmann, M. (2013). The human frontal lobes and frontal network systems: An evolutionary, clinical, and treatment perspective. ISRN Neurology, 2013. https://doi.org/10.1155/ 


\section{3/892459}

Im, C.-H. (2018). Computational EEG analysis and applications. Methods and applications. In Springer (Vol. 10, Issue 4).

Imperatori, C., Brunetti, R., Farina, B., Speranza, A. M., Losurdo, A., Testani, E., Contardi, A., \& della Marca, G. (2014). Modification of EEG power spectra and EEG connectivity in autobiographical memory: A sLORETA study. Cognitive Processing, 15(3). https://doi.org/10.1007/ s10339-014-0605-5

Kiernan, J. A. (2012). Anatomy of the temporal lobe. Epilepsy Research and Treatment, 2012,1-12. https://doi.org/10.1155/ 2012/176157

Knyazev G. G. (2012). EEG delta oscillations as a correlate of basic homeostatic and motivational processes. Neuroscience and biobehavioral reviews, 36(1), 677-695. https://doi.org/ 10.1016/j.neubiorev.2011.10.002

Levine, T. R., Serota, K. B., Carey, F., \& Messer, D. (2013). Teenagers lie a lot: A further investigation into the prevalence of lying. Communication Research Reports, 30(3). https: //doi.org/10.1080/08824096.2013.806254

Lim, K. K., Friedrich, M., Radun, J., \& Jokinen, K. (2013). Lying through the eyes: detecting lies through eye movements. In Proceedings of the 6th workshop on Eye gaze in intelligent human machine interaction: gaze in multimodal interaction. 51-56. https://doi.org/10.1145/2535948.2535954

Mandal, F. B. (2014). Nonverbal communication in humans. Journal of Human Behavior in the Social Environment, 24(4). https://doi.org/10.1080/10911359.2013.831288

McLeod, S.A. (2013). Stages of memory - encoding storage and retrieval. Simply Psychology. www.simplypsychology.org/ memory.html

Nieuwenhuis, S., Elzinga, B. M., Ras, P. H., Berends, F., Duijs, P., Samara, Z., \& Slagter, H. A. (2013). Bilateral saccadic eye movements and tactile stimulation, but not auditory stimulation, enhance memory retrieval. Brain and Cognition, 81(1), 52-56. https://doi.org/10.1016/j.bandc.2012.10.003

Nikolaev, A. R., Jurica, P., Nakatani, C., Plomp, G., \& van Leeuwen, C. (2013). Visual encoding and fixation target selection in free viewing: Presaccadic brain potentials. Frontiers in Systems Neuroscience, 7, 1-12. https://doi.org/ 10.3389/fnsys.2013.00026

Nikolaev, A. R., Nakatani, C., Plomp, G., Jurica, P., \& van Leeuwen, C. (2011). Eye fixation-related potentials in free viewing identify encoding failures in change detection. NeuroImage, 56(3), 1598-1607. https://doi.org/10.1016/j. neuroimage.2011.03.021

Nikolaev, A. R., Pannasch, S., Ito, J., \& Belopolsky, A. V. (2014). Eye movement-related brain activity during perceptual and cognitive processing. Frontiers in systems neuroscience, 8, 62. https://doi.org/10.3389/fnsys.2014.00062

Nunez, P. L., \& Srinivasan, R. (2009). Electric fields of the brain: The neurophysics of EEG. In Electric Fields of the Brain: The neurophysics of EEG. https://doi.org/10.1093/ acprof:oso/9780195050387.001.0001
Ohno, K., Funase, A., Cichocki, A., \& Takumi, I. (2007). Analysis of EEG signals in memory guided saccade tasks. IFMBE Proceedings, 14(1). https://doi.org/10.1007/978-3540-36841-0_672

Parker, A., \& Dagnall, N. (2012). Effects of saccadic bilateral eye movements on memory in children and adults: An exploratory study. Brain and Cognition, 78(3),238-247. https://doi.org/10.1016/j.bandc.2012.01.007

Plöchl, M., Ossandón, J. P., \& König, P. (2012). Combining EEG and eye tracking: Identification, characterization, and correction of eye movement artifacts in electroencephalographic data. Frontiers in human neuroscience, 6, 278. https: //doi.org/10.3389/fnhum.2012.00278

Pouget, P. (2015). The cortex is in overall control of "voluntary" eye movement. Eye (Basingstoke), 29(2). https://doi.org/10. 1038/eye.2014.284

Simola, J., le Fevre, K., Torniainen, J., \& Baccino, T. (2015). Affective processing in natural scene viewing: Valence and arousal interactions in eye-fixation-related potentials. NeuroImage, 106, 21-33. https://doi.org/10.1016/ j.neuroimage.2014.11.030

Velasques, B., Machado, S., Paes, F., Bittencourt, J., Domingues, C. A., Basile, L. F., Salles, J. I., Cagy, M., Piedade, R., Arias-Carrión, O., Sack, A. T., Cheniaux, E., Nardi, A. E., \& Ribeiro, P. (2011). Hemispheric differences over frontal theta-band power discriminate between stimulus- versus memory-driven saccadic eye movement. Neuroscience Letters, 504(3), 204-208. https://doi.org/10.1016/j.neulet. 2011.09.023

Vrij, A., Granhag, P. A., \& Porter, S. (2010). Pitfalls and opportunities in nonverbal and verbal lie detection. Psychological Science in the Public Interest, Supplement, 11(3),89-121. https://doi.org/10.1177/1529100610390861

Vrij, A., Oliveira, J., Hammond, A., \& Ehrlichman, H. (2015). Saccadic eye movement rate as a cue to deceit. Journal of Applied Research in Memory and Cognition, 4(1), 15-19. https://doi.org/10.1016/j.jarmac.2014.07.005

Wan Ismail, W. O. A. S., Hanif, M., Mohamed, S. B., Hamzah, N., \& Rizman, Z. I. (2016). Human emotion detection via brain waves study by using electroencephalogram (EEG). International Journal on Advanced Science, Engineering and Information Technology, 6(6),1005-1011. https://doi.org/10. 18517/ijaseit.6.6.1072

Xiao, R., Shida-Tokeshi, J., Vanderbilt, D. L., \& Smith, B. A. (2018). Electroencephalography power and coherence changes with age and motor skill development across the first half year of life. PLoS ONE, 13(1). https://doi.org/10.1371/ journal.pone.0190276

Yonelinas A. P. (2013). The hippocampus supports highresolution binding in the service of perception, working memory and long-term memory. Behavioural brain research, 254, 34-44. https://doi.org/10.1016/j.bbr.2013.05.030

Zlotnik, G., \& Vansintjan, A. (2019). Memory: An extended definition. Frontiers in Psychology, 10. https://doi.org/10. 3389/fpsyg.2019.02523 